\title{
Dimensional interaction in hyperactive children: Classification of computer-displayed stimuli
}

\author{
MARY ANN FISHER \\ University of Maryland Baltimore County, Baltimore, Maryland 21228
}

\begin{abstract}
Selective attention was assessed in three groups of three hyperactive boys, aged 5-7, 7-9, and 9-11 years, from their performance on a classification task using dimensions that are separable for adults. Stimuli were presented by cathode-ray tube display. The type of dimensional interaction found in the two younger groups of hyperactive boys was similar to the type found by Shepp in about 33\% of unselected 6-year-old children: interference with orthogonal dimensions, relative to single dimensions, and interference with correlated dimensions only for the easier single dimensions. This type of dimensional interaction is not among the types considered perceptually meaningful in Garner's classification system. These results suggest that the younger boys processed the stimuli dimensionally but with a failure of selective attention.
\end{abstract}

Separable and integral are two major types of dimensional interactions described by Garner (e.g., 1976), and related by him to different perceptual processes. One way in which separable dimension pairs are distinguished from integral dimension pairs is by the different effects of dimensional combinations on speeded classification of stimuli. Subjects are asked to sort decks of cards according to values of one of the dimensions. The decks vary in the relationship of the values of the target dimension to the values of the nontarget dimension. In the unidimensional deck, only the (usually two) values of the target dimension vary from card to card, with a neutral value or no value of the nontarget dimension present on every card. In the correlated dimensions deck, the values of the target dimension are correlated with the values of the nontarget dimension. In the orthogonal deck, all combinations of the values of the target and nontarget dimensions appear and are uncorrelated. A dimension pair is integral if the correlated sort is faster than the unidimensional sort and the orthogonal sort is slower than the unidimensional sort. A dimension pair is separable if there is no difference in the sorting times for unidimensional, correlated, and orthogonal decks.

Dimension pairs which are separable for adults progress from integral, at about $6 \mathrm{yrs}$, to separable, at about 9 yrs, for unselected children (Shepp \& Swartz, 1976; Shepp, Note 1). Additional evidence that children progress from integral to separable processing comes from a study by Strutt, Anderson, and Well (1975). Comparing

The author notes with gratitude the helpful comments of Marilyn D. Wang in conversations throughout the course of this investigation. Ramona Roberts deserves thanks for running subjects. The University of Maryland Computer Science Center provided the computer facilities for data analysis. Reprint requests should be sent to: Mary Ann Fisher, Department of Psychology, University of Maryland Baltimore County, 5401 Wilkens Avenue, Baltimore, Maryland 21228. only orthogonal to unidimensional sorts, Strutt et al. found interference with orthogonality which decreased in magnitude with increasing chronological age (CA). Strutt et al. point out that instructions to respond quickly are necessary to produce interference with orthogonal dimensions when the card-sorting task is used.

The method of classification, adapted to cathode-ray tube (CRT) display of stimuli to be classified, is used in the study presented here. This method has several advantages over the card-sorting technique. Data collection is automatic and not subject to the same experimenter bias as the card sort, which requires stopwatch timing. Timing includes only the interval from the presentation of the stimulus to the classification response, so that time taken for processes that occur outside this interval may be separated out. Errors and response times for errors are identified. In addition, children tend to respond quickly without special speed instructions. Because of these advantages, this technique may prove useful in a further delineation of perceptual processes occurring in classification tasks using card sorts.

\section{METHOD}

\begin{abstract}
Subjects
Children were randomly selected on the basis of age from a group of boys accepted to a hospital study unit for evaluation and treatment of hyperactive behavior. Three boys in each of three CA groups, 5-7, 7-9, and 9-11 years were tested. The children were accepted to the study group on the basis of an outside physician's clinical impression of hyperactivity and, for those in school, outside teachers' ratings of at least one standard deviation above the norm established for boys by Werry, Sprague, and Cohen (1975) on Factor II (attention deficit) and Factor IV (hyperactivity) of Conner's Teacher Rating Scale. The children were hospitalized during the evaluation period. Clinical impressions of physicians and hospital staff, and ratings by the in-house teacher on the Conner scale were also used to distinguish hyperactivity from other behavior disorders. As might be
\end{abstract}


expected, these judgments were not always concordant. In particular, the in-house teacher's ratings of the behavior of these children in the small hospital class tended to be lower than the outside teacher's ratings. In several cases the in-house teacher's ratings were not different from the means for the Werry, Sprague, and Cohen (1975) norms. This may indicate the situational nature of the hyperactivity, the adaptation level of the inhouse teacher, or the heterogeneous nature of the group with respect to kind of behavior disorder. The children were participating in a drug study to evaluate treatment with dextroamphetamine and were given placebo on the test day. At least 1 drugfree day intervened between amphetamine and placebo test days.

\section{Apparatus and stimuli}

Stimuli were presented to the child, one at a time, on a Tektronix Type 611 storage display unit, with the longer side horizontal, set at intensity level 2.2 , placed on a table with the display at eye level approximagely $40 \mathrm{~cm}$ in front of the seated child. The child turned on the CRT stimulus display and started the trial timer by pressing the middle key of three $1.25-\mathrm{cm}$ square keys, $2.5 \mathrm{~cm}$ from center to center of side-by-side keys, on a key set placed on the table between the child and the CRT. The outer two buttons were marked with the appropriate values of the target dimension. The child judged which of the two cue values was displayed and pressed the appropriate key stopping the trial timer. Response time was recorded in ticks $(1 / 60 \mathrm{sec})$. Stimulus presentation and response time recording were controlled by a dedicated PDP 11/40.

The three dimensions were: (1) Dot position. A dot, $5 \mathrm{~mm}$ in diam, was displayed either at the top, center of the dot at CRT coordinates $(511,542)$ or at the bottom $(511,225)$ of the screen. (2) Number of lines. One vertical line $4 \mathrm{~cm}$ long, midpoint at $(511,383)$, or two such lines $5 \mathrm{~mm}$ apart on either side of the $x=512$ vertical at $x=503$ and $x=519$. (3) Form. A rectangle, corners $(383,511),(639,511),(639,255)$, and $(383,255)$, or a diamond, corners $(511,511),(639,383),(511,255)$, and $(383,383)$.

The maximal extent of the displayed figure was approximately $4.5 \times 5.5 \mathrm{~cm}$. Figure 1 is a representation of examples of the stimuli used.

\section{Procedure}

A session consisted of 270 trials. Each of the nine possible information-dimension combinations was presented in a 30 -trial

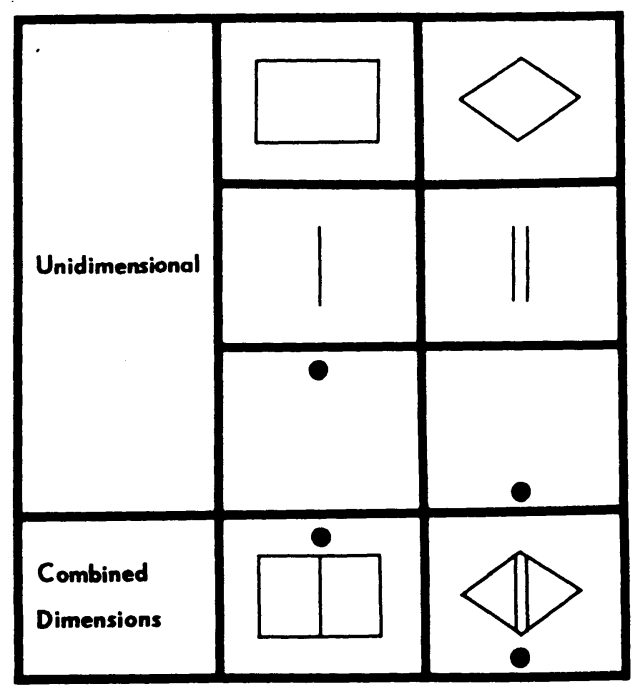

Figure 1. Examples of stimuli used. block. Three orders counterbalanced the order of information conditions within an age group, UCO, COU, and OUC, where $\mathrm{U}$, $\mathrm{C}$, and $\mathrm{O}$ are the unidimensional, correlated, and orthogonal conditions, respectively. The order of dimensions was randomly assigned DLF or FLD, where D, L, and F are the dot, line, and form dimensions, respectively. Information conditions were presented within dimensions.

Children were brought individually into the experimental room. The function of the keyboard was explained and each child was told that the first few trials would be practice trials. The child was not asked to respond quickly since it appeared from pilot work that this request often led children to disregard the display and engage in fast random keypressing. The child was also told that it was important to "try to be correct" but that "most people make a mistake once in a while and that is nothing to worry about." Any question the child asked during the first few trials of a condition was answered and the instructions were repeated if necessary. After the session the child was permitted to play computer games for a few minutes. Most children were enthusiastic about the task and all remained on task most of the time.

\section{RESULTS}

\section{Adult Performance}

The three dimensions were established to be separable for adults. Three normal adult volunteers, who were otherwise unconnected with the project, served as subjects. The sessions were run exactly as they were for the children and the order of information conditions was counterbalanced as they were in each CA group of children. Means for the adults for the three information levels were identical, $.44 \mathrm{sec}$, with no differences attributable to information level or dimension.

\section{Performance Measure}

The performance measure used for analysis was the logarithm of the response time on a correct trial of the last 25 trials, given that the trial was preceded by at least two correct trials. Geometric means for blocks of five trials were used. As a conservative measure, only correct trials that were preceded by at least two correct trials were included, since performance on error trials tends to be faster, and performance on the first correct trial following an error tends to be slower, than that on the remaining correct trials in the block. Arithmetic mean response times were $1.56,2.55$, and $2.08 \mathrm{sec}$ for errors, correct trials following errors, and remaining correct trials within the same block, respectively $[F(2,186)=10.64$, $\mathrm{p}<.01$, for the overall comparison, $\mathrm{F}(1,93)=16.51$, $\mathrm{p}<.01$, for errors compared with corrects following corrects, and $F(1,93)=4.14, p<.05$, for corrects following errors compared with other corrects]. The probabilities of an error on a trial were $.10, .05$, and .04 for Groups 5-7, 7-9, and 9-11, respectively, and .06, .04, and .08 for the unidimensional, correlated, and orthogonal conditions, respectively. These differences did not reach statistical significance.

The first five trials of each information-dimension condition were considered warm-up or practice trials. 
The first trial was usually very long regardless of condition, and instructions were occasionally repeated to the child during these trials.

To limit the occasional very long and very short correct trials, the analyses repeated here were redone with an upper $(2.5-\mathrm{sec})$ and lower $(.5-\mathrm{sec})$ limit imposed on the trials. The results of the analyses with this additional restriction were consistent with the analyses reported here. The principal effect of the imposition of the additional restriction was to increase slightly the significance of the effects.

\section{Chronological Age Groups}

Geometric means are reported here throughout, and those for each information-dimension combination for each CA group are presented in Table 1. In all analyses of variance, information (unidimensional, correlated, and orthogonal), dimension (dot, line, form), and blocks (last five blocks of five trials each) were repeatedmeasures factors and subjects were nested within groups. The group means were $1.74,1.41$, and $1.04 \mathrm{sec}$ for Groups $5-7,7-9$, and 9-11, respectively $[F(2,6)=3.14$, $\mathrm{p}>.10]$. The differences among information conditions, $1.22,1.39$, and $1.53 \mathrm{sec}$ for the unidimensional, correlated, and orthogonal conditions, respectively, were marginally significant $[F(2,12)=2.80, p>.10]$.

There was a significant Information by Groups interaction $[F(4,12)=3.44, p<.05]$. When groups were considered separately, the Information by Groups interaction resulted from a significant difference due to information condition in Group 5.7 $[F(2,4)=7.95$, $\mathrm{p}<.05]$, a marginally significant effect of information $[F(2,4)=5.10, p<.08]$ for Group $7-9$, and no significant main effects or interactions with information in Group 9-11.

\section{Comparisons Among Information-Dimension Conditions}

Shepp (Note 1) found about 33\% of the 6-year-old children had a redundancy gain with the more difficult dimension and a redundancy loss with the easier dimension. Since it appeared that the younger hyperactive

Table 1

Geometric Mean Response Time (in Seconds) for InformationDimension Combinations for Three Chronological Age Groups

\begin{tabular}{clrrr}
\hline \multirow{2}{*}{ Group } & & \multicolumn{3}{c}{ Dimension } \\
\cline { 3 - 5 } & & Dot & Line & Form \\
\hline \multirow{4}{*}{$5-7$ Years } & Unidimensional & 1.66 & 1.50 & 1.33 \\
& Correlated & 1.58 & 1.83 & 1.80 \\
& Orthogonal & 2.31 & 1.99 & 1.91 \\
& Unidimensional & 1.52 & 1.12 & .98 \\
7-9 Years & Correlated & 1.58 & 1.29 & 1.25 \\
& Orthogonal & 1.85 & 1.63 & 1.78 \\
& Unidimensional & 1.10 & 1.01 & .94 \\
9-11 Years & Correlated & 1.16 & 1.10 & 1.07 \\
& Orthogonal & .91 & .98 & 1.07 \\
\hline
\end{tabular}

children had a similar pattern, and since the two easier dimensions, line and form, were indistinguishable from each other and each distinguishable from the more difficult dot dimension, two kinds of analyses were performed, one for the dot dimension and one which included both line and form. For the dot dimension, there were no effects of information or age that reached significance.

In the analyses for line and form there was no significant main effect of dimension or interaction involving dimension. There was a difference due to information level with means of $1.13,1.36$, and $1.50 \mathrm{sec}$ for the unidimensional, correlated, and orthogonal conditions, respectively $[F(2,12)=11.80, p<.01]$. There was also a marginally significant interaction of Groups by Information, reflecting the decreasing effect of orthogonality with increasing age $[F(4,12)=2.66, .05<p<.10]$. Comparisons of pairs of information conditions showed that, for line and form, performance in the unidimensional condition was significantly faster than performance in the correlated condition $[F(1,6)=21.80, p<$ $.01]$, and unidimensional was significantly faster than orthogonal $[F(1,6)=17.80, p<.01]$. In the comparison of the correlated and orthogonal conditions, the difference between conditions did not reach significance $[\mathrm{F}(1,6)=2.24, .10<\mathrm{p}<.25]$.

\section{DISCUSSION}

The response patterns of this group of hyperactive boys do not match any of the patterns considered perceptually meaningful in Garner's (1976) classification of types of dimensional interaction. The primary characteristic that distinguishes the performance of these children is the relationship between performances in the unidimensional and correlated conditions. Although performance on the relatively difficult dot dimension was not slower in the correlated condition relative to the single dimension condition, performance on the two easier dimensions, line and form, was slower in the correlated condition. Dimensions that interact to produce slower performance in the correlated condition relative to the single dimension condition are not among the types related by Garner to perceptual processes. These may, however, play some role in children's perception. Shepp (Note 1), for instance, reported similar findings of interference with easier dimensions in the correlated condition in about $33 \%$ of the 6 -year-old children he studied.

For the younger hyperactive boys, the dot, line, and form dimensions, when combined, appear to form a compound from which it is difficult to extract dimensional information. There are at least three processes that might account for this effect. (1) The dimensions are processed integrally and the increased dissimilarity of the key-markings to the stimulus presented on the CRT accounts for the slower performance in the correlated condition. This explanation is made less plausible by the fact that the slower performance in the correlated condition occurred only for the two dimensions of line and form. There is no indication that the performance on the dot dimension is also slower, as would be required if this explanation were to obtain. (2) The emergent compounds are processed as unrelated entities for which two classification responses must be learned. In this case, the increased difficulty of the orthogonal over the correlated condition would arise from the presence of eight, rather than two, entities whose names must be learned. This explana- 
tion is made less plausible by the several facts that the child has only unidimensional representations of the cues of the target dimension on the response keys, that the child receives no feedback on the correctness of his responses, that the child makes very few errors in any condition, and that the child often announces immediately that he has "made a mistake" and shows the aftereffects of an error by taking more time on the following correct trial. Virtually errorless learning of two or eight compounds without feedback and without extracting dimensional information is highly improbable. (3) In the combined stimulus, dimensions are processed exhaustively before the decision on the basis of the target dimension is made. While this principle alone can account for the asymmetric interference with correlated dimensions, it does not account for the additional time required for processing the stimuli in the orthogonal set.

An interpretation of the additional interference is suggested by some of Garner's (1976) comments on sources of interference. Garner points out that in the few cases in which interference with correlated dimensions has been observed (such as in the Stroop effect), response competition, rather than perceptual process, is involved. It is unlikely, however, that response competition can account for the interference with correlated dimensons for these hyperactive children for two reasons. The first is that the labels for the values of the dimensions are not in obvious conflict (rectangle, one line, dot-on-top as opposed to diamond, two lines, dot-on-bottom). The second is that the response keys, which were correlated with dimensional values, were not in conflict in the correlated condition; each of the three values presented in combination in the correlated condition was associated with the same key when its dimension was the target. Although response competition can be ruled out as the source of interference in the correlated dimensions condition, it cannot be ruled out as the source of the additional interference in the orthogonal dimensions condition, in which the correlation of response keys and values of the nontarget dimensions was zero.

A plausible account of the pattern of behavior of hyperactive children is, therefore, that the failure of selective attention with correlated dimensions arises from the combined dimensions being processed exhaustively, and that the additional interference with orthogonal dimensions arises from response competition.

\section{REFERENCE NOTE}

1. Shepp. B. E. From perceived similarity to dimensional structure: A new hypothesis about perceptual development. Paper prepared for SSRC Conference on The Nature of Human Categorization, Lake Arrowhead, California, May 26-29, 1976.

\section{REFERENCES}

GARNER, W. R. Interaction of stimulus dimensions in concept and choice processes. Cognitive Psychology, 1976, 8. 98-123.

Shepp, B. E., \& Schwartz, K. B. Selective attention and the processing of integral and nonintegral dimensions: A developmental study. Journal of Experimental Child Psychology, 1976, 22. 73-85.

Strutt, G. F.. Anderson, D. R., \& Well, A. D. A developmental study of the effects of irrelevant information on speeded classification. Journal of Experimental Child Psychology, 1975, 20, 127-135.

Werry, J. S., Sprague, R. L., \& Cohen, M. N. Conner's teacher rating scale for use in drug studies with children: An empirical study. Journal of Abnormal Child Psychology, 1975, 3, 217-229.

(Received for publication August 1, 1977.) 